\title{
Neutrosophic Triangular Fuzzy Travelling Salesman Problem Based on Dhouib-Matrix- TSP1 Heuristic
}

\author{
(On Neutrosophic Triangular Fuzzy Travelling Salesman Problem)
}

\author{
Souhail Dhouib \\ Department of Industrial Management, \\ Higher Institute of Industrial Management, \\ University of Sfax, Tunisia \\ Email: souhail.dhouib [AT] gmail.com
}

\begin{abstract}
In this paper, the Travelling Salesman Problem is considered in neutrosophic environment which is more realistic in real-world industries. In fact, the distances between cities in the Travelling Salesman Problem are presented as neutrosophic triangular fuzzy number. This problem is solved in two steps: At first, the Yager's ranking function is applied to convert the neutrosophic triangular fuzzy number to neutrosophic number then to generate the crisp number. At second, the heuristic Dhouib-Matrix-TSP1 is used to solve this problem. A numerical test example on neutrosophic triangular fuzzy environment shows that, by the use of Dhouib-Matrix-TSP1 heuristic, the optimal or a near optimal solution as well as the crisp and fuzzy total cost can be reached.
\end{abstract}

Keywords-component; Neutrosophic Number; Combinatorial Optimization; Travelling Salesman Problem; Triangular fuzzy; Yager's Ranking function; Dhouib-Matrix-TSP1 Heuristic.

\section{INTRODUCTION}

The philosophy of neutrosophic was introduced in 1995 by Smarandache [1]. It consists of neutrosophic: set, logic probability, statistics and theory (solving problems with indeterminacy in many fields of sciences). Three independent membership functions are enhanced in a neutrosophic set: the Truth $(T)$, the Indeterminacy $(I)$ and the Falsity $(F)$ where $T, I$ and $F$ are subsets of $]^{-} 0,1^{+}[$.

Recently, the neutrosophic concept has become a focus of several research papers such as the resolution of the Travelling Salesman Problem (TSP) under neutrosophic domain through the Ones Assignment method [2] and the Branch and Bound technique [3]. The optimization of the Assignment Problem in triangular fuzzy neutrosophic environment using the Branch and Bound technique and the efficiency is illustrated on a realworld agricultural problem in [4]. Furthermore, the resolution of the neutrosophic trapezoidal Assignment Problem via a novel method [5] and the measurement of the credit risk degree for a private Bank is carried out through the development of a neutrosophic Analytical Hierarchy Process model in [6].

The TSP is a classical problem in the field of combinatorial optimization where the main objective is to search for the shortest cycle between all cities for a salesman. In fact, the salesman needs to start from a specified city, then visit all cities only once and return to the starting city. The TSP is formulated as follows:

Minimize:

$$
\sum_{i=1}^{n} \sum_{j=1}^{n} d_{i j} x_{i j}
$$

Subject to:

$$
\begin{aligned}
& \sum_{j=1}^{n} x_{i j}=1, \quad i=1, \ldots, n \\
& \sum_{i=1}^{n} x_{i j}=1, \quad j=1, \ldots, n \\
& x_{i j}=0 \text { or } 1, \quad i=1, \ldots, n, \quad \mathrm{j}=1, \ldots, n
\end{aligned}
$$

Where the distance between city $i$ and city $j$ is denoted by $d_{i j}$ and a binary variable $x_{i j}$ is used (if city $i$ and $j$ are connected then $x_{i j}=1$ else $x_{i j}=0$ ). In this paper, we consider the TSP in neutrosophic triangular fuzzy environment in which each element $d_{i j}$ in the distance matrix is considered as a neutrosophic triangular fuzzy number. In the purpose of solving this problem, we enrich our recent heuristic DhouibMatrix-TSP1 (DM-TSP1) with the Yager's ranking function. This paper is outlined as follows: in section 2, we study the neutrosophic set concept. In section 3, we present the heuristic DM-TSP1 with Yager's ranking function. In section 4, we illustrate the resolution by applying the adapted technique DM-TSP1 on a numerical example. Finally, in section 5, we present the conclusion and our further research work. 


\section{THE NEUTROSOPHIC TRIANGULAR FUZZY NUMBER}

The triangular fuzzy number $\tilde{T}$ is denoted by $\tilde{T}=\left(t_{1}, t_{2}, t_{3}\right)$, where $t_{1}, t_{2}$ and $t_{3}$ are real numbers with $t_{1} \leq t_{2} \leq t_{3}$. Let's consider $X$ a space of points where the generic elements in $X$ are denoted by $x$. Accordingly, the membership function $\mu_{\tilde{T}}(x)$ for a triangular fuzzy number $\tilde{T}$ is defined as:

$$
\mu_{\tilde{T}}(x)=\left\{\begin{array}{lll}
\frac{x-t 1}{t 2-t 1} & \text { if } & t 1 \leq x \leq t 2 \\
1 & \text { if } \quad x \leq t 2 \\
\frac{t 3-x}{t 3-t 2} & \text { if } t 2 \leq x \leq t 3 \\
0 & \text { Otherwise }
\end{array}\right.
$$

The neutrosophic set $T^{N}$ over $X$ has the form $T^{N}=\left\{\left\langle x: T_{t^{N}}(x), I_{t^{N}}(x), F_{t^{N}}(x)\right\rangle, x \in X\right\} \quad$ where the functions $\left.T_{t^{N}}, I_{t^{N}}, F_{t^{N}}: X \rightarrow\right]^{-} 0,1^{+}[$with the condition ${ }^{-} 0 \leq T_{t^{N}}(x)+I_{t^{N}}(x)+F_{t^{N}}(x) \leq 3^{+}$.

The neutrosophic triangular fuzzy set $T^{N}=\left\{\left\langle x: T_{t^{N}}(x), I_{t^{N}}(x), F_{t^{N}}(x)\right\rangle, x \in X\right\}$, presents three neutrosophic triangular fuzzy numbers: the truth $T_{t^{N}}(x)=\left(T_{t 1^{N}}(x), T_{t 2^{N}}(x), T_{t 3^{N}}(x)\right) \quad$ the indeterminacy $I_{t^{N}}(x)=\left(I_{t 1^{N}}(x), I_{t 2^{N}}(x), I_{t 3^{N}}(x)\right)$ and the falsity $F_{t^{N}}(x)=\left(F_{t 1^{N}}(x), F_{t 2^{N}}(x), F_{t 3^{N}}(x)\right)$.

Here is an example (see Figure 1) of a graphical representation of $T^{N}=\langle(0,1,6),(0,2,8),(2,3,8)\rangle$.

\section{Neutrosophic Triangular Fuzzy TSP}

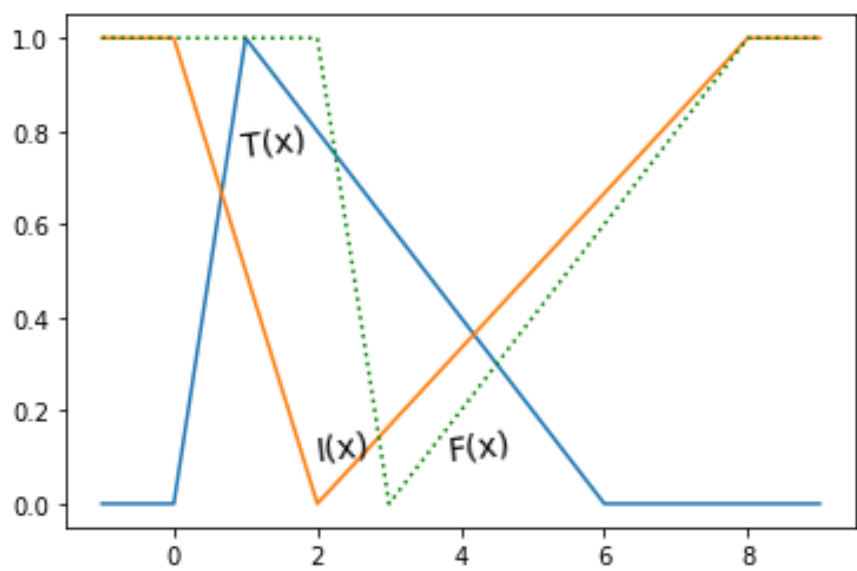

Figure 1 Graphical representation of the neutrosophic triangular fuzzy set

\section{THE HEURISTIC DHOUIB-MATRIX-TSP1: DM-TSP1}

In order to rapidly generate an initial basic feasible solution for the TSP we designed and developed in [7] a new heuristic named DM-TSP1. It is a novel constructive column-row method based on several rules. Then, we perform a stochastic version of DM-TSP1 in [8, 9] entitled DM-TSP2. Moreover, we adapted the heuristic DM-TSP1 for the case of: the triangular fuzzy TSP in [10], the trapezoidal fuzzy TSP in [11] and the octagonal fuzzy TSP in [12]. Furthermore, to solve the Transportation Problem we propose in [13] a novel deterministic heuristic entitled Dhouib-Matrix-TP1.

This paper presents the adaptation of the DM-TSP1 to solve the TSP in neutrosophic environment. The first phase consists in applying twice the Yager's function [14] in order to transform the neutrosophic triangular fuzzy set to crisp numbers (viewing that the DM-TSP1 works on crisp numbers). Let's assume $\tilde{T}=\left(t_{1}, t_{2}, t_{3}\right)$ a triangular fuzzy number, then the corresponding Yager's ranking function will be defined as:

$$
R(\tilde{T})=\int_{0}^{1} 0.5\left(t_{\alpha}^{L}, t_{\alpha}^{U}\right) d \alpha
$$

With $\left(t_{\alpha}^{L}, t_{\alpha}^{U}\right)=\left\{\left(t_{2}-t_{1}\right) \alpha+t_{1}, t_{3}-\left(t_{3}-t_{2}\right) \alpha\right\}$.

The DM-TSP1 is composed of four steps:

\section{Step 1}

- Compute the standard deviation for each row and write it on the right-hand side of the matrix. Next, find the maximum standard deviation value and select its row. Then, choose the smallest element in this row which will specify the two first cities $x$ and $y$ to be inserted in the list List-cities $\{x, y\}$. Finally, discard the respected columns of city $x$ and city $y$.

\section{Step 2}

- Find the minimal element for city $x$ and for city $y$ and select the smallest distance which will indicate city $z$.

\section{Step 3}

-Add city $z$ to the list List-cities and discard its column. Next, go to step 2 if there is not a column to be discarded otherwise go to step 4.

\section{Step 4}

-Modify the realizable solution in List-cities in order to generate a cycle (the starting city in the cycle has to be also the last one). First, to ensure that the starting city will be at the first position, translate all cities existing before the starting city (one by one) at the end of the list. Second, duplicate the starting city at the last position. 


\section{COMPUTATIONAL RESUlTS}

Let's consider the following neutrosophic triangular fuzzy TSP discussed in [2]: a company with four areas and a salesman which has to distribute products between them using the following neutrosophic fuzzy matrix cost (see Figure 2).

$$
\left(\begin{array}{llll}
\infty & d_{12} & d_{13} & d_{14} \\
d_{21} & \infty & d_{23} & d_{24} \\
d_{31} & d_{32} & \infty & d_{34} \\
d_{41} & d_{42} & d_{43} & \infty
\end{array}\right)
$$

Figure 2 The neutrosophic triangular fuzzy set matrix

Where:

$$
\begin{aligned}
& d_{12}=[(1,2,3) ;(3,4,5) ;(4,5,6)] \\
& d_{13}=[(0,1,2) ;(1,2,3) ;(2,3,4)] \\
& d_{14}=[(0,1,6) ;(0,3,6) ;(2,8,10)] \\
& d_{21}=[(0,1,2) ;(0,2,4) ;(2,3,4)] \\
& d_{23}=[(0,1,6) ;(2,4,6) ;(4,5,6)] \\
& d_{24}=[(1,2,7) ;(0,3,10) ;(2,8,10)] \\
& d_{31}=[(0,1,6) ;(0,2,8) ;(2,3,8)] \\
& d_{32}=[(0,4,8) ;(3,4,9) ;(2,8,10)] \\
& d_{34}=[(0,1,2) ;(0,2,4) ;(3,4,5)] \\
& d_{41}=[(0,2,4) ;(2,3,4) ;(4,6,8)] \\
& d_{42}=[(0,2,4) ;(1,2,7) ;(2,4,6)] \\
& d_{43}=[(0,1,2) ;(1,2,3) ;(2,3,4)]
\end{aligned}
$$

The first step is to convert the neutrosophic triangular fuzzy cost matrix to neutrosophic matrix using the Yager's ranking function (see Figure 3).

$$
\left(\begin{array}{cccc}
\infty & (2,4,5) & (1,2,3) & (2,3,7) \\
(1,2,3) & \infty & (2,4,5) & (3,4,7) \\
(2,3,4) & (4,5,7) & \infty & (1,2,4) \\
(2,3,6) & (2,3,4) & (1,2,3) & \infty
\end{array}\right)
$$

Figure 3 The neutrosophic matrix

Next, we transform the neutrosophic numbers to crisp numbers using Yager's ranking function and we obtain the corresponding matrix shown in Figure 4.

$$
\left(\begin{array}{cccc}
\infty & 3.75 & 2 & 3.75 \\
2 & \infty & 3.75 & 4.5 \\
3 & 5.25 & \infty & 2.25 \\
3.5 & 3 & 2 & \infty
\end{array}\right)
$$

Figure 4 The neutrosophic crisp matrix
Now, we can start the execution of the DM-TSP1 by computing the standard deviation for each row then selecting the highest one which is 1.88 in row 3 (see Figure 5) and find its minimal element (at position $d_{34}$ ).

$\left[\begin{array}{cccc}0 & 3.75 & 2 & 3.75 \\ 2 & 0 & 3.75 & 4.5 \\ 3 & 5.25 & 0 & 2.25 \\ 3.5 & 3 & 2 & 0\end{array}\right] \begin{aligned} & 1.55 \\ & 1.74 \\ & 1.88 \\ & 1.34\end{aligned}$

Figure 5 Select the element $d_{34}$

Add cities 3 and 4 to List-cities $\{3-4\}$ then discard columns 3 and 4 . Besides, find the minimal element in rows 3 and 4 which corresponds to value 3 in row 3 at position $d_{31}$ (see Figure 6).

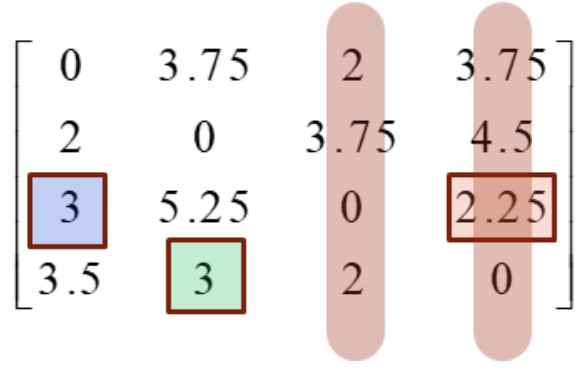

Figure 6 Discard columns 3 and 4

Next, add city 1 at the left side in List-cities $\{1-3-4\}$ viewing that it is chosen from city 3 then discard column 1 (see Figure $7)$.

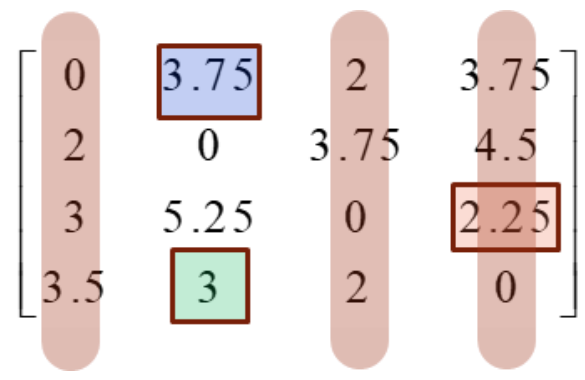

Figure 7 Discard column 1

Likewise, choose the minimal element in rows 1 and 4 which is 3 at position $d_{42}$. Now, add city 2 to List-cities $\{1-3-4-2\}$ at the right side viewing that it is created from city 4 and discard column 2 (see Figure 8).

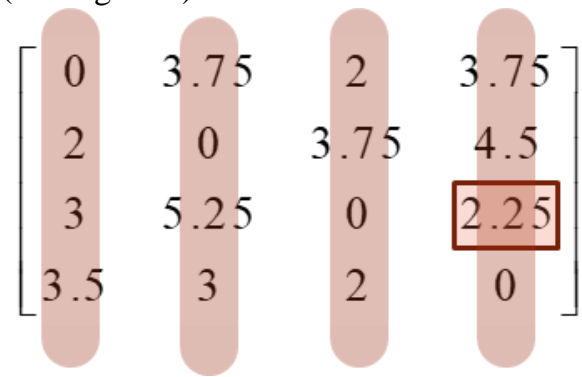

Figure 8 Discard column 2 
The last step is to create a cycle from List-cities by changing in the respected order all cities and inserting city 1 at the last position to generate the following cycle: $\{1-3-4-2-1\}$. The corresponding optimal crisp solution obtained by the heuristic DM-TSP1 using Yager's ranking function is $\{1-3-4-2-1\}=2+$ $2.25+3+2=9.25$ which corresponds to the neutrosophic optimal solution: $(1,2,3)+(1,2,4)+(2,3,4)+(1,2,3)=(5$, $9,14)$. Noting that this optimal result has been already found by [2] using the Ones Assignment method and the Pascal's graded mean ranking function.

\section{CONCLUSION}

In real-life industry, several complex problems can be represented under neutrosophic philosophy. In this paper, the Traveling Salesman Problem in neutrosophic triangular fuzzy environment is considered and solved by the means of the novel heuristic Dhouib-Matrix-TSP1 based on Yager's ranking function. To prove the efficiency of Dhouib-Matrix-TSP1 to solve neutrosophic triangular fuzzy problems a numerical case study is illustrated. Future works will deal with the resolution of the Multi-Objective Travelling Salesman Problem under the neutrosophic environment.

\section{REFERENCES}

[1] F. Smarandache, "A Unifying Field in Logics: Neutrosophic Logic. Neutrosophy, Neutrosophic Set, Neutrosophic Probability and Statistics", 2005, Infinite Study.

[2] S. Subasri and K. Selvakumari "Solving Neutrosophic Travelling Salesaman Problem in Triangular Fuzzy Number Using Ones Assignment Method", Eurasian Journal of Analytical Chemistry, 2018, vol. 13 pp. $285-291$.

[3] S. Subasri and K. Selvakumari, "Neutrosophic Travelling Salesman Problem in Trapezoidal Fuzzy number using Branch and Bound Technique", Journal of Physics: Conference Series, 2019, doi:10.1088/1742-6596/1362/1/012098.
[4] S. Krishna Prabha and S. Vimala, "Neutrosophic Assignment Problem via BnB Algorithm", Advances in Algebra and Analysis, Trends in Mathematics, 2019, pp. 323-330.

[5] H. A. E. Khalifa and P. Kumar, "A Novel Method for Neutrosophic Assignment Problem by using Interval-Valued Trapezoidal Neutrosophic Number", Neutrosophic Assignment Problem, 2020, vol. 36, pp. 24-36.

[6] N. A. Ibrahem, H. Elghareeb, F. F. Farahat and A. AboElfotouh, "Comparative Mathematical Model for Predicting of Financial Loans Default using Altman Z-Score and Neutrosophic AHP Methods", 2021, Neutrosophic Sets and Systems, vol. 43.

[7] S. Dhouib, "A New Column-Row Method for Traveling Salesman Problem: The Dhouib-Matrix-TSP1", International Journal of Recent Engineering Science, 2021, vol. 8, Issue 1, pp.6-10.

[8] S. Dhouib, "Stochastic Column-Row Method for Travelling Salesman Problem: the Dhouib-Matrix-TSP2", International Journal of Engineering Research \& Technology, 2021, vol. 10, issue 3, pp. 524527.

[9] S. Dhouib, "Minimizing the Total Distance for the Supply Chain Problem Using Dhouib-Matrix-TSP2 Method", International Journal of Advanced Research in Engineering and Technology, 2021, vol. 12, issue 5, pp. 1-12.

[10] S. Dhouib, "Haar Dhouib-Matrix-TSP1 Method to Solve Triangular Fuzzy Travelling Salesman Problem", Research Journal of Recent Sciences, vol. 10, no. 3, pp. 1-3, 2021.

[11] Sa. Dhouib and S. Dhouib, "Optimizing the Trapezoidal Fuzzy Travelling Salesman Problem Through Dhouib-Matrix-TSP1 Method Based on Magnitude Technique", International Journal of Scientific Research in Mathematical and Statistical Sciences, 2021, vol. 8, Issue 2, pp. 1-4.

[12] M. Miledi, S. Dhouib and T. Loukil, "Dhouib-Matrix-TSP1 Method to Optimize Octagonal Fuzzy Travelling Salesman Problem Using $\alpha$-Cut Technique", International Journal of Computer and Information Technology, 2021, vol. 10, Issue 3, pp. 130-133.

[13] S. Dhouib, "A Novel Heuristic for the Transportation Problem: DhouibMatrix-TP1", International Journal of Recent Engineering Science, 2021, vol. 8, Issue 4, pp. 1-5.

[14] R. R. Yager, "A procedure for ordering fuzzy subsets of the unit interval", Information Sciences, 1981, vol. 24, pp. 143-161. 